\title{
Paraprotein-related renal disease
}

Ritika Rana, Jennifer H Pinney, and Helen J Lachmann

Ritika Rana MBBS MRCP is a renal registrar at the Queen Elizabeth Hospital Birmingham, University Hospitals Birmingham NHS Foundation Trust, UK. Email: ritika.rana@uhb.nhs.uk.

Competing interests: none declared.

Jennifer H Pinney MD MRCP is a consultant nephrologist at the Queen Elizabeth Hospital Birmingham, University Hospitals Birmingham NHS Foundation Trust, UK. Email: jennifer.pinney@uhb.nhs.uk. Competing interests: none declared.

Helen J Lachmann MD FRCP FRCPath is a reader in medicine and honorary consultant nephrologist at the National Amyloidosis Centre, UCL Division of Medicine and Royal Free London NHS Foundation Trust, London, UK. Email: h.lachmann@ucl.ac.uk.

Competing interests: none declared.

\begin{abstract}
Paraprotein related renal disease represents a diverse group of rare diseases characterised by distinct renal injury due to direct or indirect effects of a nephrotoxic paraprotein, secreted by a clone of $B$ cells. Early diagnosis and use of rapidly effective chemotherapy agents have improved patient and renal outcomes for these disorders. Patients can present with proteinuric renal impairment or tubular dysfunction. Diagnosis is often challenging due to wide range of disease manifestations, difficulties with detection of pathogenic clone, and the common finding of an incidental paraprotein in the elderly. A renal biopsy along with haematological work up is required to link a paraprotein with kidney disease. Chemotherapy directed at the plasma cell clone can halt the production of the paraprotein, which in turn may benefit renal function.
\end{abstract}

\section{Keywords}

Amyloid; cast; glomerulonephritis; myeloma; monoclonal gammopathy of renal significance; paraprotein; plasma cell; serum free light chain.

\section{Word count}

3006

\section{Number of figures}

3

\section{Number of tables}




\section{Introduction}

A paraprotein is a monoclonal immunoglobulin (Mlg) or its components (light or heavy chain) produced by clonal proliferation of plasma cells, or other cells of B cell lineage. The spectrum of disease associated with a monoclonal gammopathy is wide, from overt malignancy such as multiple myeloma (MM) to the pre malignant condition monoclonal gammopathy of undetermined significance (MGUS). This latter term is used when a circulating Mlg causes no detectable tissue damage. MGUS is a relatively common finding in the elderly; with an estimated prevalence of $3 \%$ in those older than 50 years old and carries a $1 \%$ per year risk of progression.

In the majority of patients with kidney disease and a monoclonal gammopathy, the paraprotein is incidental to the renal pathology. Monoclonal gammopathy of renal significance (MGRS) is rare and refers to kidney diseases caused by a nephrotoxic paraprotein. The underlying clonal disorder is usually low grade; previously this may have been wrongly labelled as MGUS. Although the underlying clone does not cause tumour complications nor meet current haematological criteria for treatment, specific therapy aimed at the clone may be required to prevent further renal damage or slow progression to end stage renal failure (ESRF) (1).

The range of haematological conditions that can produce a nephrotoxic Mlg and result in MGRS includes smouldering MM, smouldering Waldenstrom macroglobulinemia (WM), monoclonal B cell lymphocytosis $(\mathrm{MBL})$, low grade chronic lymphocytic lymphoma (CLL) and other low grade lymphomas. Once the haematological condition progresses to requiring treatment in its own right i.e. overt MM, WM, advanced CLL or malignant lymphoma these are no longer considered MGRS and managed according to disease specific protocols (1).

MGRS-associated kidney diseases encompass a wide spectrum of renal pathology and can be categorized according to the ultrastructural characteristics of the deposits in the kidney (table 1). The innate structural and physiochemical properties of the individual light or heavy chain in combination with the renal environment determine the type of renal lesion. Lambda light chains are found in the majority of immunoglobulin light chain $(\mathrm{AL})$ amyloidosis, whereas monoclonal kappa light chains are found in three quarters of light chain deposition disease (LCDD). The Mlg can cause renal injury by intratubular cast formation as in cast nephropathy, direct tubular toxicity, or deposition in various compartments as in amyloid.

\section{Diagnosis}

Patients with unexplained urinary abnormalities (proteinuria and/or haematuria), or renal impairment should have a paraprotein screen. This includes serum protein electrophoresis with immunofixation, urine protein electrophoresis with immunofixation and serum free light chain (sFLC) analysis. 
A renal biopsy is integral to diagnosing the underlying renal lesion, which can help to guide treatment and is prognostically important. A kidney biopsy should include immunofluorescence (IF) and electron microscopy (EM) to establish monoclonality and characterize the immunoglobulin (Ig) deposit ultrastructurally.

A detailed haematologic work up (bone marrow aspirate and biopsy, flow cytometry, immunohistochemistry, FISH testing +/- lymph node biopsy) is required to correlate the specific lg found on renal biopsy with that found on haematologic work up to establish a link between paraprotein and kidney disease.

\section{Myeloma}

Multiple myeloma is a cancer of plasma cells and accounts for almost $10 \%$ of all haematological malignancies. The worldwide incidence varies from 4- 50 cases per million population per year. In the UK there are 5,500 new cases of MM per year. The median age at onset is 70 years and it is more common in men, and in African Americans.

Renal impairment is a common complication of MM with up to $50 \%$ of patients presenting with some degree of dysfunction. Approximately $20 \%$ of cases have severe renal disease classifying this as a myeloma defining event, and up to $5 \%$ have severe acute kidney injury (AKI) requiring dialysis (2); $90 \%$ of severe AKI in myeloma is due to myeloma-cast nephropathy.

The revised international myeloma working group (IMWG) diagnostic criteria for MM comprises the presence of clonal plasma cells and a myeloma defining event of one or more of the classic 'CRAB' features of: hypercalcemia, renal failure, anemia and/or destructive bone lesions, or evidence of a heavy tumor burden defined by one or more of $>60 \%$ plasma cells on bone marrow biopsy, sFLC ratio of $>100$, or $>1$ focal bone lesion on MRI scan.

Patients can present with systemic clinical features such as; weight loss, anaemia, recurrent infections, hypercalcemia and bone pain. Some patients may present with AKI, low-grade proteinuria and a bland urinary sediment.

The current overall survival from first diagnosis is 4-years and disease free survival (from first remission to relapse) is around 18 months. There has been an improvement in the past decade in overall survival for patients with MM with the introduction of new therapies.

\section{Myeloma-cast nephropathy}

Monoclonal free light chains (FLCs) aggregate with Tamm-Horsfall protein to produce casts (figure 2). Histologically they can often be seen as large, fractured or laminated casts within the distal tubule 
or collecting ducts. They are often surrounded by multinucleated giant cells and there is evidence of chronic interstitial damage. Myeloma casts cause tubular obstruction that in turn leads to AKI.

Certain conditions, such as dehydration, hypercalcaemia, sepsis, or insults from radiological contrast media or non-steroidal anti-inflammatory drugs, give FLCs a higher propensity to precipitate as casts and can worsen the renal insult.

Cast nephropathy is more common when the sFLC concentration exceeds $500 \mathrm{mg} / \mathrm{L}$. In patients presenting with severe $\mathrm{AKI}$ and evidence of a high concentration of light chains the haematologic work up should take priority to enable rapid treatment to start and a renal biopsy is not usually indicated as it will not alter management.

Severe renal impairment is an adverse determinant of outcome and survival improves if there is early recovery of kidney function. Since the introduction of bortezomib based chemotherapy and better supportive care, around $50 \%$ of patients with MM who require dialysis at presentation recover independent kidney function.

Studies have shown no benefit of plasma exchange in myeloma cast nephropathy (MCN). Two randomised controlled trials have recently been completed: MYRE and EuLITE. Both reported no difference in renal recovery at 3 months in patients treated with dialysis using high cut-off membranes (HCO-HD) compared to standard high-flux hemodialysis (HF-HD). There was an increase in overall renal recovery in the MYRE study, but not in the EuLITE study, where there was an increased mortality reported at 2-years in patients who received HCO-HD.

\section{Waldenström's macroglobulinemia}

Waldenström's macroglobulinemia is due to clonal proliferation of lymphoplasmacytic cells. Renal complications are less common than in patients with MM. Typically the renal lesion is due to intracapillary thrombi, secondary to IgM deposition, and may be associated with cryoglobulinemia. WM can also cause light chain cast nephropathy, nephrotic syndrome (due to amyloid deposition), non-amyloid nephrotic syndrome (with a minimal change-like picture). Treatment is generally rituximab based.

\section{AL amyloidosis}

Amyloidosis is a disorder of protein misfolding and refers to the extracellular deposition of low molecular weight proteins as fibrils. AL amyloidosis is due to the deposition of light chains which undergo a conformational change and are deposited in the extracellular space as amyloid fibrils. Accumulation of these fibrils causes progressive disruption to organ structure and function. 
An estimated 500-600 people are diagnosed in the UK every year. The median age at diagnosis is 64 years and it is more common in males. The clinical presentation in $\mathrm{AL}$ amyloidosis depends on number and nature of organs affected (table 1). Many patients present with non-specific symptoms such as malaise and weight loss.

The diagnosis of $\mathrm{AL}$ amyloidosis requires evidence of a monoclonal plasma cell disorder and direct demonstration of amyloid fibrils on biopsy. The presence of amyloid fibrils can be confirmed by their characteristic appearance on EM and by their ability to bind Congo red with green birefringence under polarized light. The type of protein within the amyloid fibrils can be determined by immunohistochemical staining, IF or laser microdissection with mass spectrometry. Whole body ${ }^{123}$ /-labelled serum amyloid P (SAP) scintigraphy can identify amyloid in solid organs and can be used in serial follow-up assessments to track the total body amyloid load (figure 3).

Median survival in untreated patients is only $12-15$ months depending on the organs involved. Poor prognostic factors in patients with renal amyloid include high NT-pro B-type natriuretic peptide, older age, higher total serum FLC concentration, lower serum albumin, hypotension and hyperbilirubinaemia. The degree of renal impairment, serum albumin and amount of proteinuria are all associated with progression to dialysis. Outcome on dialysis is only slightly better than in patients with myeloma but is improving over time, and patients with isolated renal involvement can do well. Renal transplantation in selected patients with sustained clonal remissions can achieve an excellent outcome and recurrence in the graft is not commonly found (3).

\section{Monoclonal immunoglobulin deposition disease (MIDD)}

MIDD is characterized by deposition of Mlgs or its components in various organs; the kidney is most commonly affected. The diagnosis differs from amyloidosis as here the light (or heavy) chain fragments do not form fibrils and deposits are Congo red negative. Three subtypes are: light chain deposition disease (LCDD), heavy chain deposition disease (HCDD), and light and heavy chain deposition disease (LHCDD). Of these LCDD is the most common accounting for $80 \%$ of cases.

LCDD is characterized by deposition of non-organized granular deposits composed of Mlg light chains along the glomerular and tubular basement membranes. The diagnosis is usually made on EM. The kidney is the principal target organ as light chains are filtered by the glomeruli, reabsorbed in proximal tubules, and degraded in tubular cells by lysosomal enzymes.

LCDD usually presents in the sixth decade, and is more common in men. Presentation is usually with hypertension and microscopic haematuria, and $50 \%$ of cases have associated nephrotic syndrome. Most cases have advanced chronic kidney disease (CKD), ESRF occurs in up to $30 \%$. LCDD is 
associated with kappa light chains in $80 \%$ of cases and more than $60 \%$ have underlying myeloma. Extrarenal manifestations of LCDD are seen in approximately $30 \%$ of cases, with cardiac infiltration occurring in $20 \%$ and clinically overt liver involvement in $10-20 \%$. There have also been reports of LCDD in gastrointestinal tract and skin biopsies but this is extremely rare.

LCDD has a better outcome than other paraprotein-related renal diseases with a median patient survival of 7 years. LCDD should be aggressively treated with chemotherapy as achieving a deep clonal response prolongs renal survival, and prevents allograft failure from recurrent LCDD in those who receive renal transplants (4). Extra-renal involvement and lack of clonal response to chemotherapy are both features of a poor prognosis.

\section{Other paraprotein-related renal diseases}

Fibrillary glomerulonephritis is a glomerular disease characterized by Congo red negative fibrils that are randomly arranged and larger than amyloid fibrils. Presentation is similar to LCDD with $50 \%$ of patients presenting with nephrotic range proteinuria. Haematuria and hypertension are also common features.

There is some evidence that it is more common in the context of malignancy, dysproteinaemias and autoimmune disease. There are very limited data regarding response to treatment and the optimal management of this condition has yet to be established.

Immunotactoid glomerulonephritis is rare; fibrils are larger, usually greater than $30 \mathrm{~nm}$ in diameter and arranged in parallel microtubules. There can be an underlying lymphoplasmacytic disorder.

In Light chain proximal tubulopathy (LCPT) light chains form localised crystals or inclusions within the proximal tubules. Patients present with Fanconi syndrome (e.g. aminoaciduria, normoglycemic glycosuria, hypophosphatemia, hypouricemia, sub nephrotic-range proteinuria). In patients who have significant renal impairment and/or proteinuria, treatment with clone directed chemotherapy seems a reasonable approach.

Proliferative glomerulonephritis with monoclonal immunoglobulin deposits (PGNMID) mimics an immune complex glomerulonephritis. The most common pattern is a membranoproliferative glomerulonephritis (MPGN). Immunofluorescence reveals monotypic deposits and 20\% patients have a detectable paraprotein. Patients with MPGN on light microscopy should be evaluated for an underlying clone.

C3 glomerulopathy with monoclonal gammopathy is characterized by lack of renal Mlg deposits, although $60-80 \%$ patients over 50 years have a clone at the time of diagnosis. Here the Mlg acts as a C3 nephritic factor or anti factor $\mathrm{H}$ antibody and results in uncontrolled activation of alternative complement 
pathway. Observational studies support targeted chemotherapy if a clone is detected or based upon the isotype of the circulating monoclonal protein detected in the serum or urine.

\section{General treatment principles}

Treatment of paraprotein related kidney disease indicated to preserve or restore kidney function and prevent recurrence after kidney transplantation. The choice of chemotherapy regimen depends on the nature of the underlying clone i.e. lymphocytic or plasmacytic in origin. The renal response strongly correlates with the nature of the hematologic response and rapid suppression of Mlg secretion with chemotherapy is required to improve outcomes. Effective treatment involves disease specific management and supportive care.

\section{Chemotherapy}

The aim of treatment is to rapidly suppress paraprotein production, producing a deep and sustained clonal response while minimizing treatment-related mortality and morbidity. Response to treatment is assessed by measuring the change in the sFLC and paraprotein concentrations.

The choice of chemotherapy depends on the underlying haematological diagnosis, the degree of renal failure and/or other organ involvement and local availability of therapeutic agents. In patients with MM who are unsuitable for a stem cell transplant, first line treatment is usually a combination of either thalidomide or bortezomib with an alkylating agent and corticosteroids.

\section{General management}

The most common cause of death is sepsis; patients on chemotherapy require prophylaxis to reduce the risk of bacterial and viral infections. Prompt treatment of suspected infection is vital. In patients with proteinuria scrupulous attention must be paid to salt and water balance, and maintenance of circulating volume. Hypercalcaemia is a common cause of renal impairment in patients with MM, it should be treated promptly with hydration and bisphosphonates. Elective surgery and general anaesthesia are best avoided, and care must be taken to avoid exposure to potentially nephrotoxic drugs particularly analgesics, contrast media and antimicrobials.

\section{Preservation and replacement of organ function}

Dialysis requirement has a major impact on both survival and quality of life; however patient outcomes are improving. The responses to chemotherapy in dialysis patients is equivalent to dialysis independent, but chemotherapy can be difficult in ESRF with a higher incidence of adverse effects, even with novel agents. 
MGRS can recur frequently and sometimes rapidly after kidney transplantation, and therefore achieving a complete hematologic remission is recommended prior to transplantation. 


\section{Key points}

- Patients with unexplained proteinuria or renal dysfunction should be screened for monoclonal protein; including serum protein electrophoresis and serum and urinary free light chain assays.

- A monoclonal gammopathy can produce renal disease even with a very low level paraprotein.

- Paraprotein related renal diseases can present as AKI, progressive proteinuric renal disease, and/or Fanconi syndrome.

- The majority of elderly patients with renal impairment and MGUS have unrelated kidney disease.

- Prompt commencement of chemotherapy is crucial in MM and AKI, and patients with MGRS usually require chemotherapy.

\section{TEST YOURSELF}

To test your knowledge based on the article you have just read, please complete self-assessment questions.

Question 1

A 62 year old gentleman was referred to nephrology with an albumin creatinine ratio (ACR) of 387 $\mathrm{mg} / \mathrm{mmol}$, stable eGFR of $59 \mathrm{ml} / \mathrm{min}$, and an IgG lambda paraprotein on immunofixation, clonal $\mathrm{lg}$ quantitation was $16.17 \mathrm{~g} / \mathrm{L}$. Serum Ig levels were normal; sFLC results showed evidence of FLC clonality with $\mathrm{k}$ light chain of $20.6 \mathrm{mg} / \mathrm{l}, \lambda$ light chain of $290 \mathrm{mg} / \mathrm{l}$ and $\mathrm{a} \mathrm{k}: \lambda$ ratio of 0.071 . His haemoglobin and calcium was within normal range. A soluble immunology (ANA, ANCA) screen was negative, renal ultrasound was unremarkable, and a skeletal survey showed no bony lesion. His past medical history included a diagnosis of type 2 diabetes (recent HbA1C-IFCC 42).

What are the differential diagnoses?
A. AL amyloidosis
B. MGRS
C. Diabetic nephropathy
D. All of the above.

Answer D: All of the above.

Feedback; A bone marrow biopsy and renal biopsy are required to establish the underlying diagnosis. The key is to correlate the specific Mlg found in the kidney with the circulating paraprotein to ensure a direct link between the monoclonal gammopathy and renal lesion is established. 


\section{Question 2}

A 67 year old African American man presented with AKI stage 3 (MDRD eGFR 3ml/min, Creatinine 1500 micromol/l) requiring emergency dialysis. Haemoglobin was $81 \mathrm{~g} / \mathrm{L}$ (haematinics normal), calcium was normal. Urinalysis showed 2+protein and 3+ blood, ACR $11.3 \mathrm{mg} / \mathrm{mmol}$. A renal tract USS was normal. An IgA lambda paraprotein was present on immunofixation, clonal immunoglobulin quantitation was 26 g/L. Serum Ig showed immuneparesis (IgG $2.04 \mathrm{~g} / \mathrm{L}$, IgA $27 \mathrm{~g} / \mathrm{L}$, IgM $0.02 \mathrm{~g} / \mathrm{L}$ ), sFLC results showed evidence of free light chain excess with $\mathrm{K}$ light chain of $15 \mathrm{mg} / \mathrm{l}, \lambda$ light chain of $5835 \mathrm{mg} / \mathrm{l}$ and a $\mathrm{k}: \lambda$ ratio of 0.003. ANCA, ANA and complement levels were normal.

A bone marrow trephine biopsy showed neoplastic plasma cells accounting for $60-70 \%$ of the cellularity, and skeletal survey did not reveal any lytic lesion. He was diagnosed with IgA lambda myeloma.

What is the single most important determinant of renal recovery in MM patients with myeloma cast nephropathy?
A. Prompt start of chemotherapy
B. Extracorporeal removal of serum free light chains
C. Prompt renal replacement therapy
D. None of the above

Answer A: Prompt start of chemotherapy.

Feedback; Prompt commencement of disease specific therapy is the single most important factor in the management of patients with MM. Bortezomib based chemotherapy is first line and should be commenced as soon as possible. The renal response depends on the quality of the haematologic response, and rapid suppression of Mlg secretion with chemotherapy improves outcomes. Plasma exchange is not effective and there is not sufficient evidence of benefit to support the use of HCO-HD in routine clinical practice. 


\section{Question 3}

What are the indications for a kidney biopsy in patients with suspected paraprotein related kidney disease?
A. Progressive proteinuric CKD
B. AKI with non-light chain proteinuria.
C. Involved sFLC $<500 \mathrm{mg} / \mathrm{L}$
D. All of the above

Answer D: All of the above

Feedback; A kidney biopsy is indicated in the setting of a progressive proteinuric CKD or AKI with non-light chain proteinuria. Also if the involved sFLC $<500 \mathrm{mg} / \mathrm{L}$, as the likelihood of myeloma cast nephropathy is less and an alternative diagnosis such as MGRS may be missed. 


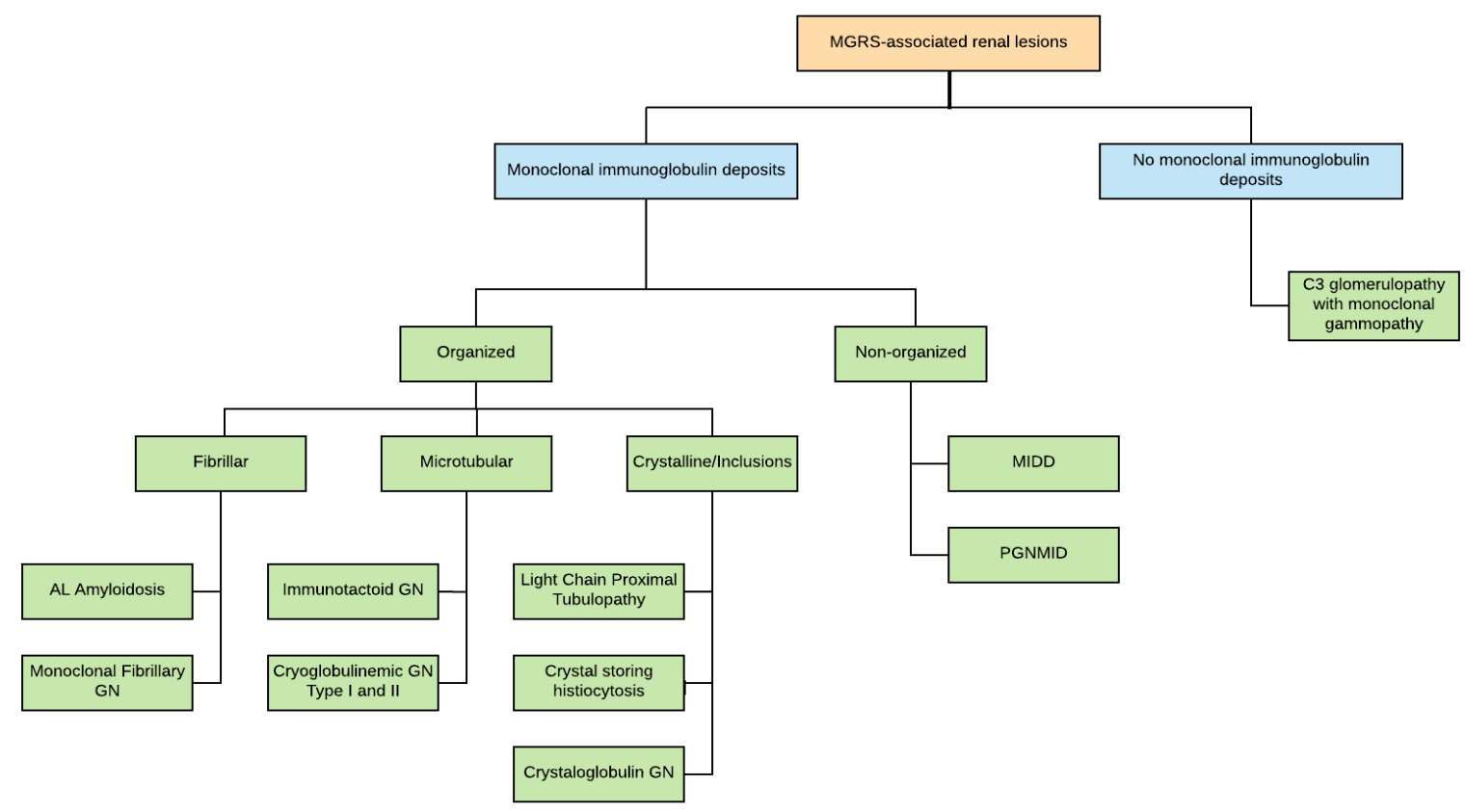

Figure 1: Classification of MGRS associated renal lesions based on the ultrastructural appearance of deposits. Adapted from Leung et al., 2019 (1).

$A L$, immunoglobulin light chain amyloidosis; GN, glomerulonephritis; MIDD, monoclonal immunoglobulin deposition disease; PGNMID, proliferative glomerulonephritis with monoclonal immunoglobulin deposits. 
Table 1.

Clinical manifestations of AL amyloidosis

Visible tissue infiltration ( $10 \%$ of cases)

Easy bruising (often periorbital)

Macroglossia with altered taste and dry mouth

Thyroid and salivary gland enlargement

Muscle and joint pseudo-hypertrophy

Autonomic neuropathy ( $15 \%$ of cases)

Orthostatic hypotension

Cardiac arrhythmias

Diarrhoea

Erectile dysfunction and impaired bladder emptying

Renal $(70 \%$ of cases)

Progressive chronic kidney disease

Proteinuria
Peripheral neuropathy (20\% of cases)

Carpal tunnel syndrome

Symmetrical distal axonal sensorimotor neuropathy

\section{Gastrointestinal}

Weight loss, anorexia, bloating

Blood loss

Constipation

Low limb voltages and pseudo-infarct pattern on ECG

\section{Hepatic}

Hepatomegaly

Increased ALP and GGT

Hepatic failure (rare)

\section{Pulmonary}

Recurrent pleural effusions

Interstitial lung involvement is usually

asymptomatic

\section{Adrenal axis}

Biochemical hypoadrenalism (rare)

AL, amyloid light chain; ALP, alkaline phosphatase; GGT, y-glutamyltransferase.

Lymphoreticular system

Splenomegaly, features of hyposplenism

Lymphadenopathy 


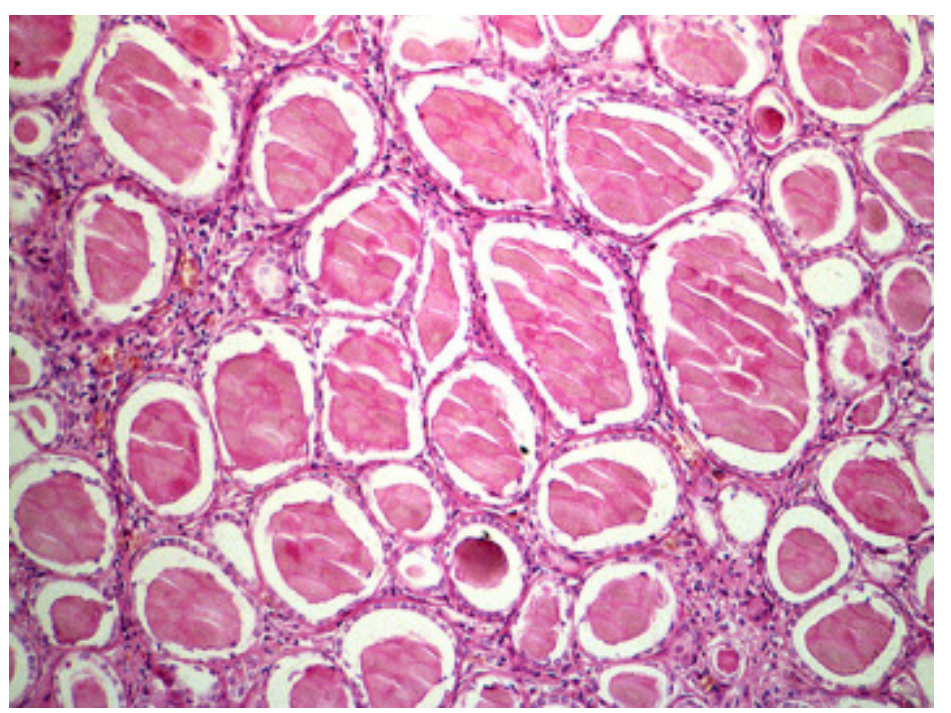

Figure 2: Myeloma kidney: multiple casts within distal tubules exhibiting fractured planes on $\mathrm{H}$ and $\mathrm{E}$ staining. 


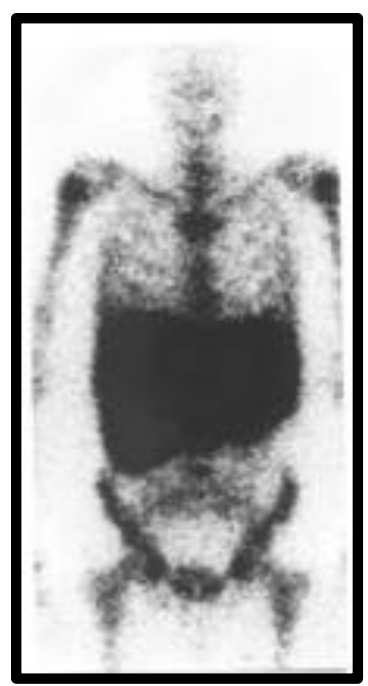

Figure 3: Anterior whole body scintigraphic image following injection of ${ }^{123}$-human serum amyloid $\mathrm{P}$ in a patient with $\mathrm{AL}$ amyloidosis. Uptake is seen in the bones, liver and spleen. 


\section{REFERENCES}

1. Leung N, Bridoux F, Batuman V, Chaidos A, Cockwell P, D'Agati VD, et al. The evaluation of monoclonal gammopathy of renal significance: a consensus report of the International Kidney and Monoclonal Gammopathy Research Group. Nature Reviews Nephrology. 2019;15(1):45-59.

2. Evison F, Sangha J, Yadav P, Aung YS, Sharif A, Pinney JA, et al. A population-based study of the impact of dialysis on mortality in multiple myeloma. British journal of haematology. 2018;180(4):588-91.

3. Pinney J, Lachmann H, Sattianayagam P, Gibbs S, Wechalekar A, Venner C, et al. Renal transplantation in systemic amyloidosis-importance of amyloid fibril type and precursor protein abundance. American Journal of Transplantation. 2013;13(2):433-41.

4. Sayed RH, Wechalekar AD, Gilbertson JA, Bass P, Mahmood S, Sachchithanantham S, et al. Natural history and outcome of light chain deposition disease. Blood. 2015;126(26):2805-10. 